\title{
Approximating Bending Stiffness for Structural Optimization of Double-skin Hollowed Car Body Panels
}

\author{
Yoshiki Ohta ${ }^{\mathrm{a}, *}$ \\ aDepartment of Mechanical Engineering, Faculty of Engineering, Hokkaido University of Science. Email: ohta@ hus.ac.jp
}

\begin{abstract}
This paper presents the evaluation of the approximate equivalent bending stiffness of double-skin hollowed car body panels. For this purpose, the equivalent bending stiffness of the rectangular panel is expressed first in the quadratic polynomial form with respect to the design parameters for structural optimization by using the Response Surface Method (RSM). Finite element formulation for bending problem of the panel is also formulated by using the ACM rectangular element, and then FE source code is developed by incorporating the equivalent stiffness obtained by the RSM. Finally, the numerical results obtained from the present FEA with the equivalent stiffness are compared with the ones by a commercial FE software, ANSYS, and then the applicability of the approximate equivalent stiffness is studied.
\end{abstract}

Keywords: Bending stiffness; double-skin hollowed extrusion; finite element analysis; response surface; structural optimization

\section{Introduction}

Aluminum alloy hollowed extrusions have been developed for a few decades, and many applications of the extrusions can be found not only in buildings and bridges but also in mechanical elements such as a railway car body and so on. In near futures it could be extensively applied to car body according to the light weightiness and the flexibility for structural optimization. Double-skin hollowed extrusions generally consist of two skins and inner ribs between the skins, and thus the topology optimization for the locations of the inner ribs and the size optimization for both of the skins and the inner ribs can be found for the design problem of the structures.

In the research for the aluminum hollowed extrusions, Kawasaki et al. [1] studied the structural FEM analysis on railway car bodies made of aluminum hollowed extrusions under uniformly distributed normal load, where the equivalent orthogonal anisotropic plate is employed through the selection of four main rigidities which describe principal deformation of car body. The FEM results calculated with orthogonal anisotropic plates are found to be in good agreement with the load test result. Kawasaki et al. also developed not only the energy absorber for crashworthy structure by using annealed aluminum hollowed extrusion

\footnotetext{
${ }^{*}$ Corresponding author. Tel.: +81-11-688-2284

Maeda 7-15-4-1, Teine-ku, Sapporo

Hokkaido, Japan, 006-8585
}

[2], but also the friction stir welding (FSW) to manufacture railway car body made of aluminum hollowed extrusions [3].

Figure 1 shows an example of a railway car body made of aluminum hollowed extrusions [4]. In the structural analysis of the railway car body, whole car body is usually divided into many double-skin hollow panels, and finite element analysis is carried out for the panel. However the computational cost for numerical evaluations would not be small due to the complexity of the structure, and thus the costs would be greater due to many iterations of numerical calculation in optimization process for the numerical structural optimization. So if the double-skin panel could be replaced in numerical calculations by an orthogonal anisotropic plate with equivalent bending stiffness, the computational cost could be reduced greatly in actual structural optimization.

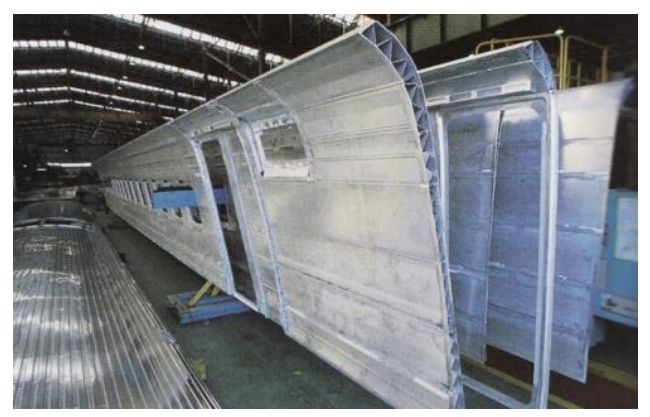

Figure 1. A railway car body made of aluminum hollowed extrusions [4] 
This paper presents the evaluations of approximate equivalent bending stiffness for the structural optimization of double-skin hollowed rectangular panels. For this purpose, the equivalent bending stiffness of the panal is expressed first in the quadratic polynomial form with respect to the design parameters, which would be taken in the definition of the optimization problem at the next stage of this work; thickness of the whole plate, thickness ratios of a skin and an inner rib, and pitch ratio of the inner rib of double-skin hollowed extrusion. Experimental points for constructing the response surface $[5,6]$ of approximate equivalent bending stiffness is calculated by using the addin software for Microsoft Excel ${ }^{\circledR}$ named RS-Maker [7]. Responses at each experimental point are calculated numerically for some simple problems such as 4-points bending problem and so on by using a commercial FE software, ANSYS. Finally, the response surfaces of the bending stiffness are determined from the responses by using the RS-Maker.

In the present paper, in order to study the applicability of the approximate equivalent bending stiffness determined, a finite element source code is also developed by introducing ACM's rectangular element and by incorporating the approximate bending stiffness obtained by the Response Surface Method, and finally numerical results obtained from the developed source code are compared with the numerical ones obtained by using ANSYS for the some numerical examples with different design parameter values.

\section{Equivalent Stiffness of Double-Skin Hollowed Panel}

Figure 2 shows the dimensions and coordinates of a double-skin hollowed rectangular panels. In the present study inner ribs are uniformly located in the $y$-direction as shown in the figure, and the widths of the plates, the pitch of an inner rib, the thickness of a skin and an inner rib and the whole thickness of the plate are denoted by $a, b, p, t$ and $h$, respectively.

At the next stage of this work a structural optimization problem will be considered for the double-skin hollowed square panel $(a=b)$, thus the whole thickness $h$, the pitch to the thickness $p / h$ and the skin thickness to the whole thickness $t / h$ are taken as design variables in the present problem. In the numerical examples, the design variables are discretized to select experimental points for response surfaces of approximate equivalent stiffness as shown in Table 1 . The width of the plate $a$ is also given such that the number of set of inner ribs is kept to be 14 in each numerical design.

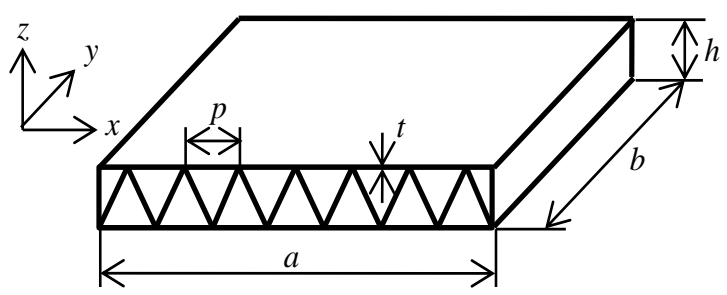

Figure 2. Dimensions and coordinates for a double-skin hollowed panel
Table 1. Discretized design variables for the double-skin panel

\begin{tabular}{ccc}
\hline$h[\mathrm{~m}]$ & $p / h$ & $t / h$ \\
\hline 0.02 & 0.8 & 0.06 \\
\hline 0.03 & 1.2 & 0.07 \\
\hline 0.04 & 1.6 & 0.08 \\
\hline 0.05 & 2.0 & 0.09 \\
\hline 0.06 & 2.4 & 0.10 \\
\hline
\end{tabular}

For the double-skin hollowed rectangular panel, bending and twisting moments $\left(M_{x}, M_{y}\right.$ and $\left.M_{x y}\right)$ can be written by using bending and twisting curvatures $\left(\chi_{x}, \chi_{y}\right.$ and $\left.\chi_{x y}\right)$ as

$$
\left\{\begin{array}{l}
M_{x} \\
M_{y} \\
M_{x y}
\end{array}\right\}=\left[\begin{array}{ccc}
D_{11} & D_{12} & 0 \\
D_{21} & D_{22} & 0 \\
0 & 0 & D_{66}
\end{array}\right]\left\{\begin{array}{l}
\chi_{x} \\
\chi_{y} \\
\chi_{x y}
\end{array}\right\}
$$

In the next section, the above stiffness $\left(D_{11}, D_{12}, D_{21}, D_{22}\right.$ and $\left.D_{66}\right)$ are tried to be expressed with respect to the design variables $(h, p / h$ and $t / h)$ by using Response Surface Methodology.

\section{Construction of Response Surfaces for the Stiffness}

In the present study, each response surface for the equivalent bending stiffness is expressed by using a quadratic polynomial as follows:

$$
\begin{aligned}
y\left(x_{1}, x_{2}, x_{3}\right)= & \beta_{0}+\beta_{1} x_{1}+\beta_{2} x_{2}+\beta_{3} x_{3} \\
& +\beta_{4} x_{1}^{2}+\beta_{5} x_{2}^{2}+\beta_{6} x_{3}^{2} \\
& +\beta_{1} x_{1} x_{2}+\beta_{1} x_{1} x_{3}+\beta_{1} x_{2} x_{3}
\end{aligned}
$$

where $\beta_{\mathrm{i}}$ are unknown coefficients of the response surface, and the response $y$ and the variables $x_{\mathrm{i}}$ correspond to each equivalent bending stiffness $D_{i j}$ and design variables mentioned in the previous section, respectively. It is known generally that more than twice number of experimental points is required as the number of unknown coefficients, so twenty experimental points are selected in the present study under the $D$-optimum criteria by using the add-in software for Microsoft Excel® named RS-Maker [7]. Table 2 shows the design variable values for twenty experimental points obtained numerically.

Table 2. Experimental points in RSM obtained by RS-Maker $\left(x_{1}=h, x_{2}=p / h, x_{3}=t / h\right)$

\begin{tabular}{ccccccc}
\hline$x_{1}$ & $x_{2}$ & $x_{3}$ & & $x_{1}$ & $x_{2}$ & $x_{3}$ \\
\cline { 1 - 4 } \cline { 5 - 7 } 0.02 & 0.8 & 0.06 & & 0.04 & 2.4 & 0.06 \\
0.02 & 0.8 & 0.08 & & 0.04 & 2.4 & 0.10 \\
0.02 & 0.8 & 0.10 & & 0.06 & 0.8 & 0.06 \\
0.02 & 1.6 & 0.06 & & 0.06 & 0.8 & 0.08 \\
0.02 & 1.6 & 0.10 & & 0.06 & 0.8 & 0.10 \\
0.02 & 2.4 & 0.06 & & 0.06 & 1.6 & 0.06 \\
0.02 & 2.4 & 0.08 & & 0.06 & 1.6 & 0.10 \\
0.02 & 2.4 & 0.10 & & 0.06 & 2.4 & 0.06 \\
0.04 & 0.8 & 0.06 & & 0.06 & 2.4 & 0.08 \\
0.04 & 1.6 & 0.08 & & 0.06 & 2.4 & 0.10 \\
\hline
\end{tabular}




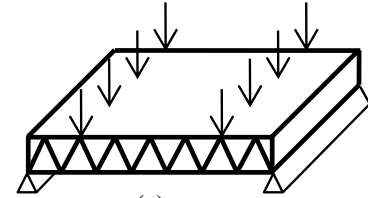

(a)

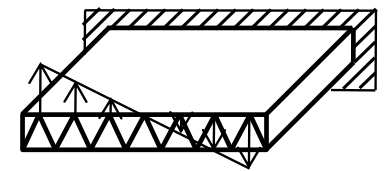

(c)

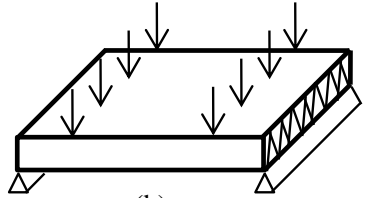

(b)

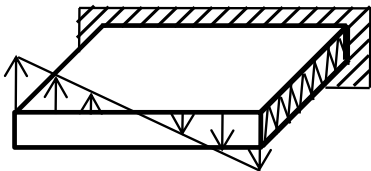

(d)
Figure 3. Numerical models employed for the evaluation of responses by using ANSYS

Table 3. Numerical conditions for finite element analysis

\begin{tabular}{cc}
\hline Software & ANSYS ED 9.0 \\
Element type & SHELL 63 \\
Young's moduls & $72 \mathrm{GPa}$ \\
Poisson's rotio & 0.33 \\
\hline
\end{tabular}

Next the response value for each experimental point, which corresponds to an equivalent stiffness value, is required in order to construct the response surfaces. In the present study, the responses are estimated from the numerical results obtained by using a commercial finite element software ANSYS for some simple bending and twisting problems shown in Fig. 3. Table 3 shows the numerical conditions for finite element analysis, the element-type employed in numerical calculations and material constants. After obtaining the responses for each experimental point by using ANSYS, each response surface is constructed for the bending stiffness by the RS-Maker.

Figure 4 shows the comparisons of stiffness values $D_{11}$ at experimental points, which are obtained by finite element analysis with ANSYS and estimated by the response surface constructed, and Figure 5 corresponds to the case of the stiffness $D_{22}$. Table 4 also shows the coefficients of determination adjusted for degree of freedom. All of the coefficients are over $98 \%$, and thus the response surfaces constructed in this study give good estimations for the equivalent bending stiffness.

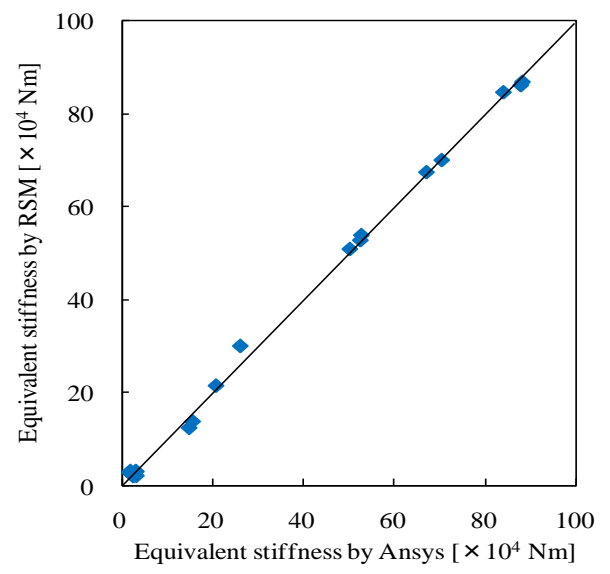

Figure 4. Comparisons of equivalent stiffness $D_{I I}$

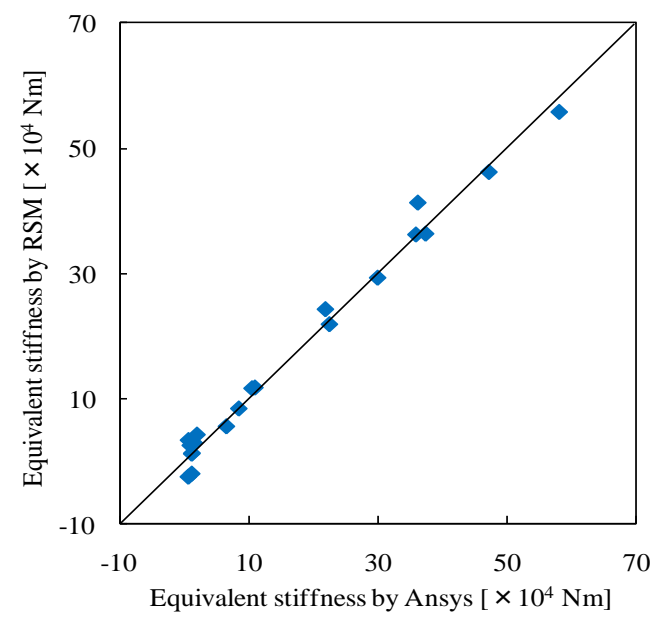

Figure 5. Comparisons of equivalent stiffness $D_{22}$

Table 4. Coefficients of determination adjusted for degrees of freedom

\begin{tabular}{cc}
\hline Stiffness & $\mathrm{R}^{2}$ \\
\hline D11 & 0.997 \\
D12 & 0.992 \\
D21 & 0.983 \\
D22 & 0.976 \\
D66 & 0.97 \\
\hline
\end{tabular}

\section{Finite Element Analysis with Response Surfaces for Bending Problem}

In the present study, the finite element source code with ACM rectangular bending element is developed by incorporating the response surfaces obtained in previous section. The applicability of the approximate bending stiffness estimated in this paper is then studied by comparing the present finite element solutions with the ANSYS's solutions where all of the skins and inner ribs are meshed for a simply bending problem of double-skin hollowed panels.

In the formulation of ACM rectangular bending element, coordinates, dimensions and nodal numbers are taken for the rectangular panel as shown in Figure 6. By using the nodal displacements $\{U\}$ and the nodal forces $\{F\}$, the governing equation is written as follows:

$$
[K]\{U\}=\{F\}
$$

where $[K]$ is the ACM stiffness matrix, and $[K]$ is calculated by

$$
[K]=\int_{-B}^{B} \int_{-A}^{A}[B]^{T}[D][B] d X d Y
$$




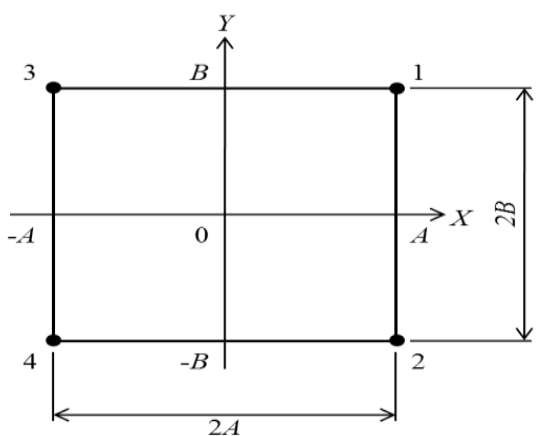

Figure 6. An ACM bending element

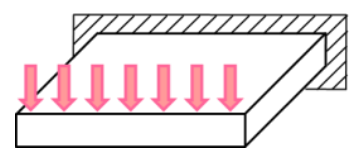

(a) Plate model by FEM code

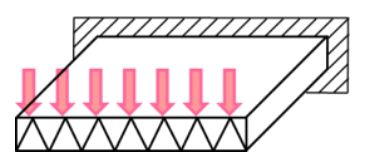

(b) Hollow extrusion model by ANSYS
Figure 7 Numerical examples

In the present study, the matrix $[D]$ is calculated by using the response surfaces for equivalent bending stiffness constructed in the previous section, and matrix $[B]$ is determined from the ACM formulations.

In numerical examples, a cantilevered panel is picked up, and uniformly distributed loads is considered as shown in Fig. 7.

Figure 8 shows the comparisons of the maximum deflections of the panel with different inner-rib thickness ratios, which is one of design variables in present problem, obtained by present FE source code and by the ANSYS. Figs. 9 and 10 also correspond to the results for the plate with different pitch ratios and inner-rib thicknesses, respectively. In each case, there are good agreements qualitatively except for some cases of small thickness values around between 0.02 and 0.03 in Fig. 10.

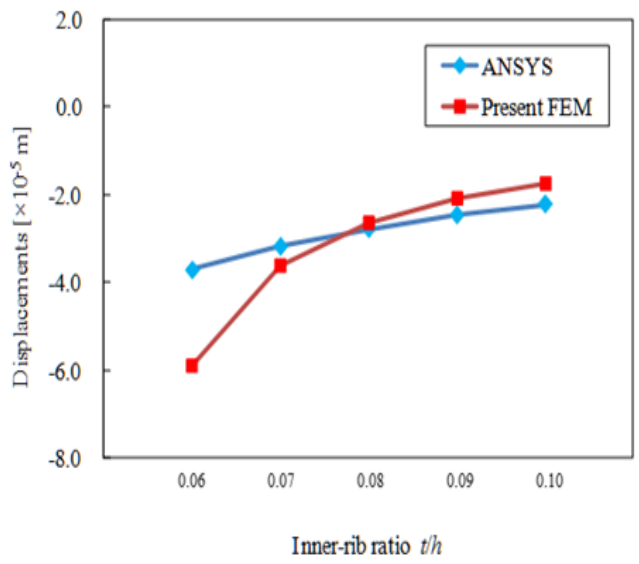

Figure 8. Evaluations of equivalent stiffness in the present study $(h=0.04, p / h=1.6)$

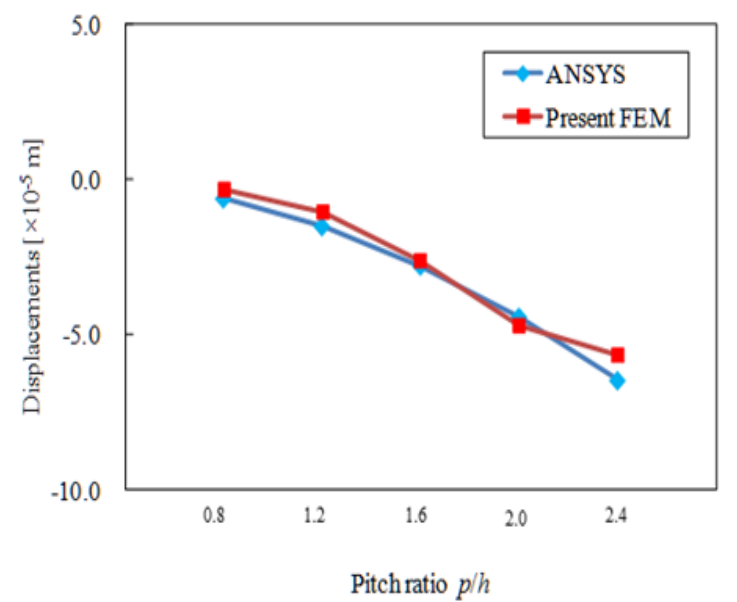

Figure 9. Evaluations of equivalent stiffness in the present study $(h=0.04, t / h=0.08)$

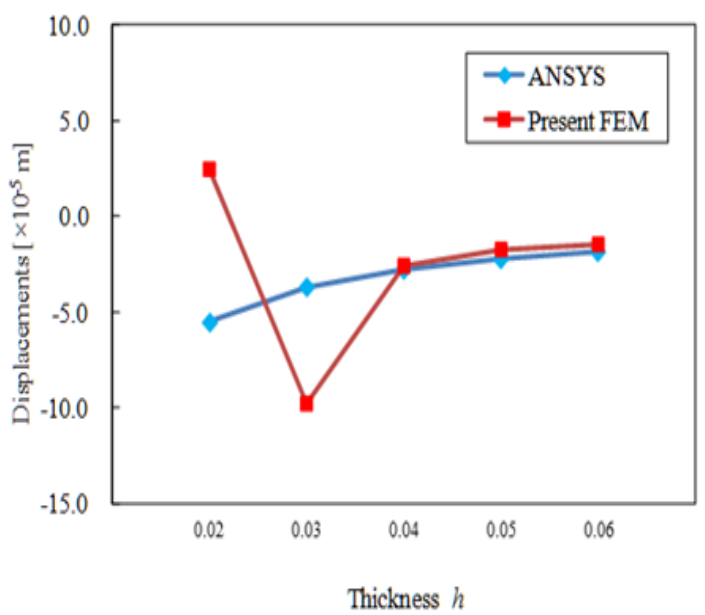

Figure 10. Evaluations of equivalent stiffness in the present study $(p / h=1.6, t / h=0.08)$

\section{Conclusions}

In the present paper, the response surfaces for the equivalent bending stiffness of a double-skin hollowed rectangular panel are first obtained with respect to parameters of dimensions of the plate by using Response Surface Methodology. In order to study the applicability of the approximate bending stiffness estimated, a finite element source code is developed by incorporating the approximate bending stiffness obtained, and then numerical results obtained by the source code developed are compared with the numerical ones obtained by using ANSYS. In the numerical examples there are good agreements qualitatively except for some exceptions, and it can be finally concluded that the present evaluation method for the equivalent bending stiffness of double-skin hollowed plates would be effective to reduce the computational cost in optimization calculations after some improving. 


\section{References}

[1] Takeshi K., Sumio O., Kentarou M., Kazufumi Y., Tomomichi O. Ryouji I. and Shinichi H, Structural Analysis of Railway Car Body of Aluminium Hollow Extrusions [in Japanese], Transaction of JSME (Ser. A), 65-636, 1999, pp.1832-1838.

[2] Takeshi K., Takahisa Y., Hideshi O., Kentarou M. and Hideyuki N., Development of Energy Absorber Using Annealed Aluminium Hollow Extrusions for Railway Vehicles [in Japanese], Transaction of JSME (Ser. A), 70-697, 2004, pp.1341-1347.

[3] Takeshi K., Toshihisa Y., Yoshihiko I., Masakuni E. and Hideyuki N., Application of Friction Stir Welding to a Railway Car Body Made of
Aluminium Hollow [in Japanese], Transaction of JSME (Ser. A), No. 71-701, 2005, pp.170-176.

[4] Takeshi H., Zusetu Shinkansen-Zenshi Vol.2 [in Japanese], GakusyuKenkyu-sya, 2005.

[5] Raymond H. Myers and Douglas C. Montgomery, Response Surface Methodology Process and Product Optimization Using Designed Experiments, Wiley Series in Probability and Statistics, John Wiley \& Sons. Inc., 1995.

[6] Douglas C. Montgomery, Design and Analysis of Experiments 4th edition, John Wiley \& Sons. Inc., 1995.

[7] Akira T., RSMaker for Excel, http://www.todoroki-lab.net /ssoft/soft.html. 